\title{
SUPER-HEDGING AMERICAN OPTIONS WITH SEMI-STATIC TRADING STRATEGIES UNDER MODEL UNCERTAINTY
}

\author{
ERHAN BAYRAKTAR AND ZHOU ZHOU
}

\begin{abstract}
We consider the super-hedging price of an American option in a discrete-time market in which stocks are available for dynamic trading and European options are available for static trading. We show that the super-hedging price $\pi$ is given by the supremum over the prices of the American option under randomized models. That is, $\pi=\sup _{\left(c_{i}, Q_{i}\right)_{i}} \sum_{i} c_{i} \phi^{Q_{i}}$, where $c_{i} \in \mathbb{R}_{+}$ and the martingale measure $Q^{i}$ are chosen such that $\sum_{i} c_{i}=1$ and $\sum_{i} c_{i} Q_{i}$ prices the European options correctly, and $\phi^{Q_{i}}$ is the price of the American option under the model $Q_{i}$. Our result generalizes the example given in Hobson \& Neuberger (2016) that the highest model based price can be considered as a randomization over models.
\end{abstract}

\section{INTRODUCTION}

Recently, using very different techniques, Bayraktar et al. (2015) and Neuberger (2007) (finally published as Hobson \& Neuberger (2017)) both calculated the super-hedging prices of American options when one is allowed to use semi-static trading strategies. In Bayraktar et al. (2015), the authors show that the super-hedging price (hedger's price) can be strictly greater than the highest model based price $\sup _{Q} \phi^{Q}$ (Nature's price), while in Hobson \& Neuberger (2017), the authors show that these two prices are equal. The reason of the difference lies in that, in Bayraktar et al. (2015) the hedger and Nature have the same information/filtration, while in Hobson \& Neuberger (2017) Nature has access to more information (i.e., Nature's filtration is larger than hedger's). As a result the definition of highest model price in the two papers are different (although the superhedging prices are the same). See Hobson \& Neuberger (2016) for another comparison of the two results. Hobson \& Neuberger (2016) also gives an example in which the highest model based price can be considered as a randomization over models.

In this paper, we show that the super-hedging price $\pi$ is given by supremum over randomized model based prices of the American option. That is, $\pi=\sup _{\left(c_{i}, Q_{i}\right)_{i}} \sum_{i} c_{i} \phi^{Q_{i}}$, where $c_{i} \in \mathbb{R}_{+}$and the martingale measure $Q^{i}$ are chosen such that $\sum_{i} c_{i}=1$ and $\sum_{i} c_{i} Q_{i}$ prices the European options correctly, and $\phi^{Q_{i}}$ is the price of the American option under the model $Q_{i}$.

Our result gives another representation of the super-hedging duality in Bayraktar et al. (2015) and builds a connection with the main result of Hobson \& Neuberger (2017). Moreover, from our result we improve on the result in Hobson \& Neuberger (2017). As indicated by our result, in terms of Nature's pricing, only the randomized models (see Definition 3.2) are relevant, and all the

Key words and phrases. American options, super-hedging, model uncertainty, semi-static trading strategies, randomized models.

E. Bayraktar is supported in part by the National Science Foundation under grant DMS-1613170 and the Susan M. Smith chair. 
other models proposed in Hobson \& Neuberger (2017) are redundant. Our result also generalizes the example provided in Hobson \& Neuberger (2016) that the highest model based price can be considered as a randomization over models.

After we wrote this note, Deng and Tan wrote a related paper, Deng \& Tan $(2016)$, in which they showed the super-hedging price equals the supremum of the expectation of some related payoff over a suitable family of martingale measures. Their approach is to enlarge the probability space by the exercise time of the American option. The main result in our paper in contrast does not require the enlargement of the space. Let us also mention that even though both paper uses the terminology "randomization", it has totally different meanings. In Deng \& Tan (2016), randomization refers to the enlarged space, while in our paper it refers to mixing the martingale measures in the original space. The related problem of hedging path dependent options using American style options is considered in Bayraktar \& Zhou (2016a), Bayraktar \& Zhou (2016b).

The paper is organized as follows. In the next section, we provide the setup and the main result. In Section 3, we give a discussion for the results in Bayraktar et al. (2015), Hobson \& Neuberger (2017), and this paper. Finally, we provide the proof for the main result in Section 4.

\section{Setup AND MAIn RESUlt}

We use the set-up in Bouchard \& Nutz (2015) and Bayraktar et al. (2015). Let $T \in \mathbb{N}$ be the time horizon and let $\Omega_{1}$ be a Polish space. For $t \in\{0,1, \ldots, T\}$, let $\Omega_{t}:=\Omega_{1}^{t}$ be the $t$-fold Cartesian product, with the convention that $\Omega_{0}$ is a singleton. We denote by $\mathcal{F}_{t}$ the universal completion of $\mathcal{B}\left(\Omega_{t}\right)$ and write $(\Omega, \mathcal{F})$ for $\left(\Omega_{T}, \mathcal{F}_{T}\right)$. Denote $\mathbb{F}:=\left(\mathcal{F}_{t}\right)_{t=0, \ldots, T}$. Let $\mathfrak{P}\left(\Omega_{1}\right)$ be the set of all the probability measures on $\left(\Omega_{1}, \mathcal{B}\left(\Omega_{1}\right)\right)$. For each $t \in\{0, \ldots, T-1\}$ and $\omega \in \Omega_{t}$, we are given a nonempty convex set $\mathcal{P}_{t}(\omega) \subset \mathfrak{P}\left(\Omega_{1}\right)$ of probability measures. We assume that for each $t$, the graph of $\mathcal{P}_{t}$ is analytic, which ensures that $\mathcal{P}_{t}$ admits a universally measurable selector, i.e., a universally measurable kernel $P_{t}: \Omega_{t} \rightarrow \mathfrak{P}\left(\Omega_{t}\right)$ such that $P_{t}(\omega) \in \mathcal{P}_{t}(\omega)$ for all $\omega \in \Omega_{t}$. Let

$$
\mathcal{P}:=\left\{P_{0} \otimes \ldots \otimes P_{T-1}: P_{t}(\cdot) \in \mathcal{P}_{t}(\cdot), t=0, \ldots, T-1\right\},
$$

where each $P_{t}$ is a universally measurable selector of $\mathcal{P}_{t}$, and

$$
P_{0} \otimes \ldots \otimes P_{T-1}(A)=\int_{\Omega_{1}} \ldots \int_{\Omega_{1}} 1_{A}\left(\omega_{1}, \ldots, \omega_{T}\right) P_{T-1}\left(\omega_{1}, \ldots, \omega_{T-1} ; d \omega_{T}\right) \ldots P_{0}\left(d \omega_{1}\right), \quad A \in \Omega .
$$

Let $S_{t}: \Omega_{t} \rightarrow \mathbb{R}^{d}$ be Borel measurable, which represents the price at time $t$ of a $d$-dimensional stock $S$ that can be traded dynamically in the market. Let $g=\left(g_{1}, \ldots, g_{e}\right): \Omega \rightarrow \mathbb{R}^{e}$ be Borel measurable, representing the European options that can only be traded at the beginning at price 0 . Assume $\mathrm{NA}(\mathcal{P})$ holds, i.e, for all $(H, h) \in \mathcal{H} \times \mathbb{R}^{e}$,

$$
(H \cdot S)_{T}+h g \geq 0 \quad \mathcal{P} \text { - q.s. } \quad \text { implies } \quad(H \cdot S)_{T}+h g=0 \quad \mathcal{P}-\text { q.s. },
$$

where $\mathcal{H}$ is the set of $\mathbb{F}$-predictable processes, $(H \cdot S)_{T}:=\sum_{t=0}^{T-1} H_{t}\left(S_{t+1}-S_{t}\right)$, and $h g$ denotes the inner product of $h$ and $g$. 11 Then from (Bouchard \& Nutz 2015, FTAP), for all $P \in \mathcal{P}$, there exists

\footnotetext{
${ }^{1}$ We say that a set is $\mathcal{P}$ polar if it is $P$-null for all $P \in \mathcal{P}$. A property is said to hold $\mathcal{P}$ - quasi-surely (q.s.) if it holds outside a $\mathcal{P}$-polar set.
} 
$Q \in \mathcal{Q}$ such that $P \ll Q$, where

$$
\mathcal{Q}:=\left\{Q \text { martingale measure }{ }^{2}: E_{Q}[g]=0 \text {, and } \exists P^{\prime} \in \mathcal{P} \text {, s.t. } Q \ll P^{\prime}\right\} \text {. }
$$

For $t=0, \ldots, T$ and $\omega \in \Omega_{t}$, define

$$
\mathcal{Q}_{t}(\omega):=\left\{Q \in \mathfrak{P}\left(\Omega_{1}\right): Q \ll P \text {, for some } P \in \mathcal{P}_{t}(\omega) \text {, and } E_{Q}\left[S_{t+1}(\omega, \cdot)-S_{t}(\omega)\right]=0\right\} \text {. }
$$

By (Bouchard \& Nutz 2015, Lemma 4.8), there exists a universally measurable selector $Q_{t}$ such that $Q_{t}(\cdot) \in \mathcal{Q}_{t}(\cdot)$ on $\left\{\mathcal{Q}_{t} \neq \emptyset\right\}$. Using these selectors we define for $t \in\{0, \ldots, T-1\}$ and $\omega \in \Omega_{t}$,

$$
\mathcal{M}_{t}(\omega):=\left\{Q_{t} \otimes \ldots \otimes Q_{T-1}: Q_{i}(\omega, \cdot) \in \mathcal{Q}_{i}(\omega, \cdot) \text { on }\left\{\mathcal{Q}_{i}(\omega, \cdot) \neq \emptyset\right\}, i=t, \ldots, T-1\right\},
$$

which is similar to (2.1) but starting from time $t$ instead of time 0 . In particular $\mathcal{M}_{0}=\mathcal{M}$, where

$$
\mathcal{M}:=\{Q \text { martingale measure }: \exists P \in \mathcal{P} \text {, s.t. } Q \ll P\} .
$$

We assume that the graph of $\mathcal{M}_{t}$ is analytic, $t=0, \ldots, T-1$. A general sufficient condition for the analyticity of $\operatorname{graph}\left(\mathcal{M}_{t}\right)$ is provided in (Bayraktar et al. 2015, Proposition 1.1).

Let $\mathcal{T}$ be the set of $\mathbb{F}$-stopping times, and $\mathcal{T}_{t}$ be the set of $\mathbb{F}$-stopping times that are no less than $t$. Denote $\|\cdot\|$ for the Euclidean norm.

Let us consider an American option with pay-off stream $\Phi$. We will assume that $\Phi:\{0, \ldots, T\} \times$ $\Omega \rightarrow \mathbb{R}$ is $\mathbb{F}$-adapted. We define the super-hedging price as

$\pi(\Phi):=\inf \left\{x \in \mathbb{R}: \exists(\tilde{H}, h) \in \mathcal{H}^{\prime} \times \mathbb{R}^{e}\right.$, s.t. $x+(\tilde{H}(t) \cdot S)_{T}+h g \geq \Phi_{t}, \mathcal{P}-$ q.s., $\left.t=0, \ldots, T\right\}$,

where

$$
\mathcal{H}^{\prime}:=\left\{\tilde{H}=(H, H(0), \ldots, H(T)) \subset \mathcal{H}^{T+2}\right\}
$$

and

$$
\tilde{H}_{s}(t):=H_{s} 1_{\{s<t\}}+H_{s}(t) 1_{\{s \geq t\}}, \quad s=0, \ldots, T .
$$

Here for $\tilde{H}=(H, H(0), \ldots, H(T)) \in \mathcal{H}^{\prime}, H$ represents the strategy that the hedger use before the American option is exercised, and $H(t)$ is the strategy the hedger will use after the American option is exercised at time $t$.

We make the following standing assumption throughout the paper.

\section{Assumption 2.1.}

(1) For $t \in\{1, \ldots, T\}$ and $(\omega, P) \in \Omega_{T} \times \mathfrak{P}\left(\Omega_{T-t}\right)$, the map $(\omega, P) \mapsto \sup _{\tau \in \mathcal{T}_{t}} E_{P}\left[\Phi_{\tau}\left(\omega^{t}, \cdot\right)\right]$ is upper-semianalytic, where $\omega^{t}$ is the path of $\omega$ up to time $t$.

(2) $\sup _{Q \in \mathcal{M}} E_{Q}[\|g\| \mid]<\infty$ and $\sup _{Q \in \mathcal{M}} E_{Q}\left[\max _{0 \leq t \leq T}\left|\Phi_{t}\right|\right]<\infty$.

Remark 2.1. If $\Phi_{t}$ is lower-semicontinuous and bounded from below for $t=0, \ldots, T$, then Assumption 2.1(1) is satisfied. See (Bayraktar et al. 2015, Proposition 3.1).

Below is the super-hedging result from (Bayraktar et al. 2015, Theorem 3.1).

\footnotetext{
${ }^{2}$ That is, $Q$ satisfies $E_{Q}\left[\left|S_{t+1}\right| \mid \mathcal{F}_{t}\right]<\infty$ and $E_{Q}\left[S_{t+1} \mid \mathcal{F}_{t}\right]=S_{t}, Q$-a.s. for $t=0, \ldots, T-1$.
} 
Lemma 2.1. Let Assumption 2.1 hold. Then

$$
\pi(\Phi)=\inf _{h \in \mathbb{R}^{e}} \sup _{Q \in \mathcal{M}} \sup _{\tau \in \mathcal{T}} E_{Q}\left[\Phi_{\tau}-h g\right],
$$

Moreover, there exists $\left(H^{*}, h^{*}\right) \in \mathcal{H}^{\prime} \times \mathbb{R}^{e}$, such that

$$
\pi(\Phi)+\left(H^{*} \cdot S\right)_{T}+h^{*} g \geq \Phi_{\tau}, \mathcal{P}-q . s ., \quad \forall \tau \in \mathcal{T}
$$

In this paper, we will get another representation for 2.3 as the super-hedging duality. Below is our main result.

Theorem 2.1. Let Assumption 2.1 hold. Then

$$
\pi(\Phi)=\sup _{\left(c_{i}, Q_{i}\right)_{i}} \sum_{i} c_{i} \sup _{\tau \in \mathcal{T}} E_{Q_{i}}\left[\Phi_{\tau}\right],
$$

where the supremum is over all finite sequence $\left(c_{i}, Q_{i}\right)_{i}$, such that for each $i, c_{i} \in(0, \infty), Q_{i} \in \mathcal{M}$, $\sum_{i} c_{i}=1$ and $\sum_{i} c_{i} Q_{i} \in \mathcal{Q}$.

Remark 2.2. Let us call the dual of the super-hedging price "the price given by Nature". We can interpret (2.4) as follows. Nature randomizes the models $\left(Q_{i}\right)_{i} \subset \mathcal{M}$ (not $\subset \mathcal{Q}$ ) in such a way that it appears to the hedger that the European options $g$ are priced correctly. Moreover, the true state $i$ is revealed at the beginning only to Nature, not to the hedger.

3. Comparison between results in Bayraktar et Al. 2015), Hobson \& Neuberger (2016), Hobson \& Neuberger (2017), And Theorem 2.1

In Bayraktar et al. (2015), Hobson \& Neuberger (2016), it is pointed out that the super-pricing price (hedger's price) can be strictly greater than the highest model based price (Nature's price). That is, it is possible that

$$
\pi(\Phi)>\sup _{Q \in \mathcal{Q}} \sup _{\tau \in \mathcal{T}} E_{Q}\left[\Phi_{\tau}\right]
$$

That is it appears that there is a duality gap between the super-hedging price and the highest model price.

In (3.1), the hedger and Nature have the same power in the sense that both use the filtration (information) $\mathbb{F}$. So in order to make Nature's price (the highest model price) equal to the hedger's price, an intuitive way is to enhance the power of Nature by providing more information to Nature. Theorem 2.1 indicates that in addition to the information $\mathbb{F}$, if Nature can also use the information of the initial distribution of the possible models $\left(Q_{i}\right)_{i}$, then the hedger's price and Nature's price will be the same.

In contrast Hobson \& Neuberger (2017), Nature can have all sorts of information as long as the models appears to be consistent to the hedger (note that the hedger only knows the information generated by the stock). To be more precise, the authors of Hobson \& Neuberger (2017) call a filtered probability space $M=\left(\Omega^{\prime}, \mathbb{F}^{\prime}=\left(\mathcal{F}_{t}^{\prime}\right)_{t=0, \ldots, T}, Q^{\prime}\right)$ a consistent model if the space $\left(\Omega^{\prime}, \mathbb{F}^{\prime}\right)$ supports a stochastic process $S$ (and random vector $g$ ), $S$ is a $\left(Q^{\prime}, \mathbb{F}^{\prime}\right)$-martingale, and $E_{Q^{\prime}}[g]=0$. 
It is then shown in Hobson \& Neuberger (2017) $]^{3}$ that the hedger's price based on the filtration generated by $S$ is equal to Nature's price (the highest model price) based on all consistent models (i.e., $\sup _{M} \phi^{M}(\Phi)$, where $\phi^{M}(\Phi)$ is the price of the American option based on the model $M$ ). Due to their proof approach, they only work on the canonical filtration for the hedger, and the European options are specified to be calls (or puts, or some other equivalent forms). Let us point out that Hobson \& Neuberger (2017) has a second result, which says that the search over all probability spaces can be reduced to a search over a much simpler class of models (see (Hobson \& Neuberger 2017, Section 2.4)).

In this paper, we show that starting from Bayraktar et al. (2015) and using a (not so conventional) min-max, we can obtain the results of Hobson \& Neuberger (2017) with a simpler proof and in fact obtain a stronger result: We demonstrate below (as an application of our main result) that only a very small subclasses of extensions which we call randomized models are relevant in representing the superhedging price. We need some preparation to describe our generalization. First, as a counterpart of (Hobson \& Neuberger 2017, Definition 1), let us first provide the following definition. Below by "embedding" we mean that $\Omega$ can be regarded as a sub space of $\Omega^{\prime}$ and $\mathcal{F}_{t}$ can be regarded as a sub sigma algebra of $\mathcal{F}_{t}^{\prime}$. (See Definition 3.2 for an example.)

Definition 3.1. We say that a filtered probability space $\left(\Omega^{\prime}, \mathbb{F}^{\prime}=\left(\mathcal{F}_{t}^{\prime}\right)_{T=0, \ldots, T}, Q^{\prime}\right)$ is belongs to the set of Nature's models if

(1) The filtered space $(\Omega, \mathbb{F})$ can be embedded in $\left(\Omega^{\prime}, \mathbb{F}^{\prime}\right)$.

(2) (After embedding) $S$ is a $\left(Q^{\prime}, \mathbb{F}^{\prime}\right)$-martingale, and $E_{Q^{\prime}}[g]=0$.

(3) (After embedding) for any $A \in \mathcal{F}_{T}$, if $\sup _{P \in \mathcal{P}} P(A)=0$ then $Q^{\prime}(A)=0$.

Denote $\mathbb{M}_{n}$ to be the collection of all such models.

Remark 3.1. Here the hedger's filtration is $\mathbb{F}$, which is not necessarily the canonical filtration generated by $S$. By Definition 3.1(1), Nature can have a strictly larger filtration $\mathbb{F}^{\prime}$.

Definition 3.2. We call $\left(\Omega^{\prime}, \mathbb{F}^{\prime}=\left(\mathcal{F}_{t}\right)_{T=0, \ldots, T}, Q^{\prime}\right)$ a randomized model, if there exist $n \in \mathbb{N}$, $\left(c_{i}\right)_{i=1, \ldots, n} \subset(0, \infty)$ with $\sum_{i=1}^{n} c_{i}=1,\left(Q_{i}\right)_{i=1, \ldots, n} \subset \mathcal{M}$ with $\sum_{i=1}^{n} c_{i} E_{Q_{i}}[g]=0$, such that

(1) $\Omega^{\prime}=\Omega \times\{1, \ldots, n\}$,

(2) $\mathcal{F}_{t}^{\prime}=\mathcal{F}_{t} \otimes \mathcal{B}(\{0, \ldots, n\})$.

(3) For $i \in\{1, \ldots, n\}$, the transaction kernel $Q^{\prime}(\cdot, i)=Q_{i}(\cdot)$, and $Q^{\prime}(\{i\})=c_{i}$.

Denote $\mathbb{M}_{r}$ as the set of all randomized models.

Remark 3.2. It is easy to see that $\mathbb{M}_{r} \subset \mathbb{M}_{n}$.

\footnotetext{
3 Hobson \& Neuberger 2016) demonstrates this on an example. It first observes (3.1) and then shows that when the models are enlarged to the consistent models there is no duality gap. It seems the authors of Hobson \& Neuberger (2017) were initially not aware of Bayraktar et al. (2015) and developed their result independently with using very different techniques. Then they wrote Hobson \& Neuberger (2016) to clarify what initially may look like a contradiction since they had not observed a duality gap.
} 
Corollary 3.1. The super-hedging price defined by $(2.2)$ is given by

$$
\pi(\Phi)=\sup _{M \in \mathbb{M}_{n}} \phi^{M}(\Phi)=\sup _{M \in \mathbb{M}_{r}} \phi^{M}(\Phi),
$$

where $\phi^{M}(\Phi)$ is the price of the American option under model $M$. That is, for $M=\left(\Omega^{\prime}, \mathcal{F}^{\prime}, Q^{\prime}\right) \in$ $\mathbb{M}_{n}$

$$
\phi^{M}(\Phi):=\sup _{\tau \mathbb{F}^{\prime} \text {-stopping time }} E_{Q^{\prime}}\left[\Phi_{\tau}\right] .
$$

Proof. It is easy to show that

$$
\pi(\Phi) \geq \sup _{M \in \mathbb{M}_{n}} \phi^{M}(\Phi) \geq \sup _{M \in \mathbb{M}_{r}} \phi^{M}(\Phi)
$$

and by Theorem 2.1 we have that

$$
\pi(\Phi)=\sup _{M \in \mathbb{M}_{r}} \phi^{M}(\Phi)
$$

\section{Proof of Theorem 2.1}

Without loss of generality, we assume that any of these European options cannot be replicated by stock $S$ and other European options. That is, for any $(H, h) \in \mathcal{H} \times \mathbb{R}^{e}$,

$$
\text { if }(H \cdot S)_{T}+h g=0 \mathcal{P}-\text { q.s., } \quad \text { then } h=0 .
$$

For otherwise, we can work on the new market where the redundant European options are removed, and obviously the super-hedging price $\pi$ would be the same. Moreover, the set of the sequences $\left(c_{i}, Q_{i}\right)_{i}$ in the supremum in 2.4 would be unchanged.

We will prove that

$$
\inf _{h \in \mathbb{R}^{e}} \sup _{Q \in \mathcal{M}} \sup _{\tau \in \mathcal{T}} E_{Q}\left[\Phi_{\tau}-h g\right]=\sup _{\left(c_{i}, Q_{i}\right)} \sum_{i} c_{i} \sup _{\tau \in \mathcal{T}} E_{Q_{i}}\left[\Phi_{\tau}\right]
$$

To this end, denote $\mathcal{M}=\left(Q_{\alpha}\right)_{\alpha \in I}$. Let

$$
A:=\left\{\left(c_{\alpha}\right)_{\alpha \in I}: c_{\alpha} \in \mathbb{R}, \text { only finitely many } c_{\alpha} \neq 0\right\} .
$$

Let $d: A \times A \mapsto \mathbb{R}$,

$$
d\left(c, c^{\prime}\right):=\sum_{\alpha \in I}\left|c_{\alpha}-c_{\alpha}^{\prime}\right|, \quad c=\left(c_{\alpha}\right)_{\alpha \in I}, c^{\prime}=\left(c_{\alpha}^{\prime}\right)_{\alpha \in I} \in A .
$$

Then it is easy to see that $d$ defines a metric, and thus $(A, d)$ is Hausdorff topological vector space. Let

$$
X:=\left\{\left(c_{\alpha}\right)_{\alpha \in I} \in A: c_{\alpha} \geq 0, \sum_{\alpha} c_{\alpha}=1\right\} .
$$

Obviously, we have that

$$
\inf _{h \in \mathbb{R}^{e}} \sup _{Q \in \mathcal{M}} \sup _{\tau \in \mathcal{T}} E_{Q}\left[\Phi_{\tau}-h g\right]=\inf _{h \in \mathbb{R}^{e}} \sup _{c \in X}\left[\sum_{\alpha} c_{\alpha} \sup _{\tau \in \mathcal{T}} E_{Q_{\alpha}}\left[\Phi_{\tau}-h g\right]\right] .
$$


Now, if the inf and sup at the right hand side of 4.2 can be exchanged without changing the value, then we have that

$$
\inf _{h \in \mathbb{R}^{e}} \sup _{Q \in \mathcal{M}} \sup _{\tau \in \mathcal{T}} E_{Q}\left[\Phi_{\tau}-h g\right]=\sup _{c \in X} \inf _{h \in \mathbb{R}^{e}}\left[\sum_{\alpha} c_{\alpha} \sup _{\tau \in \mathcal{T}} E_{Q_{\alpha}}\left[\Phi_{\tau}-h g\right]\right]=\sup _{\left(c_{i}, Q_{i}\right)} \sum_{i} c_{i} \sup _{\tau \in \mathcal{T}} E_{Q_{i}}\left[\Phi_{\tau}\right],
$$

where for the second equality, we use the fact that if $\sum_{\alpha} E_{Q_{\alpha}}[g] \neq 0$, then we can push the value inside the sup inf to $-\infty$ by choosing $h$ properly.

In the rest of the proof, we will show that the sup and inf at the right hand side of 4.2 can be exchanged. Since we do not have the compactness of the underlying sets $R^{e}$ and $X$, we will apply minimax theorem (Ha 1981, Theorem 2) (we provide it the appendix as Theorem A.1.).

Let $f: X \times \mathbb{R}^{e} \mapsto \mathbb{R}$,

$$
f(c, h):=\sum_{\alpha} c_{\alpha} \sup _{\tau \in \mathcal{T}} E_{Q_{\alpha}}\left[\Phi_{\tau}-h g\right] .
$$

It is easy to see that for $c \in X$ and $h \in \mathbb{R}^{e}$, the maps $c \mapsto f(c, h)$ and $h \mapsto f(c, h)$ are linear. For $c, c^{\prime} \in X$ and $h \in \mathbb{R}^{e}$,

$$
f(c, h)-f\left(c^{\prime}, h\right) \leq \sum_{\alpha}\left|c_{\alpha}-c_{\alpha}^{\prime}\right|\left|\sup _{\tau \in \mathcal{T}} E_{Q_{\alpha}}\left[\Phi_{\tau}\right]-h E_{Q_{\alpha}}[g]\right| \leq C_{h} \sum_{\alpha}\left|c_{\alpha}-c_{\alpha}^{\prime}\right|,
$$

where

$$
C_{h}:=\sup _{Q \in \mathcal{M}} E_{Q}\left[\max _{0 \leq t \leq T}\left|\Phi_{t}\right|\right]+\|h\| \sup _{Q \in \mathcal{M}} E_{Q}\|g\|<\infty
$$

by Assumption 2.1(2). Therefore, the map $c \mapsto f(c, h)$ is continuous. Similarly, for any $h, h^{\prime} \in \mathbb{R}^{e}$ and $c \in X$,

$$
\left|f(c, h)-f\left(c, h^{\prime}\right)\right| \leq \sum_{\alpha} c_{\alpha} E_{Q_{\alpha}}\left|\left(h-h^{\prime}\right) g\right| \leq\left\|h-h^{\prime}\right\| \sup _{Q \in \mathcal{M}} E_{Q}\|g\|,
$$

which implies that the map $h \mapsto f(c, h)$ is continuous.

We claim that $0 \in \mathbb{R}^{e}$ is an interior point of the convex set $\left\{E_{Q}[g]: Q \in \mathcal{M}\right\}$. If not, then there would exist a non-zero vector $h^{*} \in \mathbb{R}^{e}$, such that $h^{*} E_{Q}[g] \leq 0$ for any $Q \in \mathcal{M}$. Then by Bouchard $\&$ Nutz 2015, superhedging theorem), the super-hedging price of $h^{*} g$ using only stock $S$ would be no greater than 0 . Moreover, there would exist $H \in \mathcal{H}$ such that

$$
(H \cdot S)_{T} \geq h^{*} g, \quad \mathcal{P} \text {-q.s.. }
$$

Then $\operatorname{NA}(\mathcal{P})$ would imply that

$$
(H \cdot S)_{T}-h^{*} g=0, \quad \mathcal{P} \text {-q.s.. }
$$

This contradicts 4.1) since $h^{*} \neq 0$.

Let $\bar{B}(r)$ be a closed ball in $\mathbb{R}^{e}$ with radius $r$ centered at the origin, where $r>0$ is chosen such that $\bar{B}(r) \subset\left\{E_{Q}[g]: Q \in \mathcal{M}\right\}$. Denote $L:=1+\sup _{Q \in \mathcal{M}} E_{Q}\left[\max _{0 \leq t \leq T} \Phi_{t}\right]<\infty$. Let $J:=\bar{B}(3 L / r)$. Since for any $h \in \partial J$, there exist $Q^{h} \in \mathcal{M}$ such that $\left\|E_{Q^{h}}[g]\right\|=r$ and $h E_{Q^{h}}[g]=-3 L$. Then for $h \in \partial J$, there exists an open ball $B\left(h, \varepsilon^{h}\right)$ with radius $\varepsilon^{h}>0$ centered at $h$, such that

$$
h^{\prime} E_{Q^{h}}[g] \leq-2 L, \quad \forall h^{\prime} \in B\left(h, \varepsilon^{h}\right) .
$$


Since $\partial J \subset \cup_{h \in \partial J} B\left(h, \varepsilon^{h}\right)$, there exists a finite set $\left(h_{i}\right)_{i=1, \ldots, n} \subset \partial J$ such that $\partial J \subset \cup_{i=1}^{n} B\left(h_{i}, \varepsilon^{h_{i}}\right)$. For $i=1, \ldots, n$, let $\alpha_{i}$ be the index of $Q^{h_{i}}$ in $\mathcal{M}=\left\{\left(Q_{\alpha}\right)_{\alpha}: \alpha \in I\right\}$. As a result the set

$$
K:=\left\{\left(c_{\alpha}\right)_{\alpha} \in X: c_{\alpha}=0 \text { if } \alpha \notin\left\{\alpha_{i}: i=1, \ldots, n\right\}\right\}
$$

is compact. For any $h \in J^{c}:=\mathbb{R}^{e} \backslash J$ there exists some $i \in\{1, \ldots, n\}$ such that $\frac{3 L / r}{\|h\|} h \in B\left(h_{i}, \varepsilon^{h_{i}}\right)$, which implies

$$
h E_{Q^{h_{i}}}[g] \leq-2 L \frac{\|h\|}{3 L / r} \leq-2 L .
$$

Therefore,

$$
\inf _{h \in J^{c}} \sup _{c \in K} f(c, h) \geq \inf _{h \in J^{c}} \sup _{c \in K} \sum_{\alpha} c_{\alpha} E_{Q_{\alpha}}[-h g]-L \geq L \geq \sup _{c \in X} f(c, 0) \geq \inf _{h \in \mathbb{R}^{e}} \sup _{c \in X} f(c, h) .
$$

Applying Theorem A.1, we have that

$$
\inf _{h \in \mathbb{R}^{e}} \sup _{c \in X} f(c, h)=\sup _{c \in X} \inf _{h \in \mathbb{R}^{e}} f(c, h),
$$

and this completes the proof.

\section{Appendix A. A nOn-COMPaCt minimax theOREM}

Below is a minimax theorem without compactness of the underlying sets from $\mathrm{Ha}(1981)$.

Theorem A.1. Let $X, Y$ be nonempty convex sets, each in a Hausdorff topological vector space, and let $f$ be a real-valued function defined on $X \times Y$ such that

(a) For each $x \in X, f(x, y)$ is lower semi-continuous and quasi-convex on $Y$;

(b) For each $y \in Y, f(x, y)$ is upper semi-continuous and quasi-concave on $X$.

If there exists a nonempty compact convex set $K$ in $X$ and a compact set $H$ in $Y$ such that

$$
\inf _{y \in Y} \sup _{x \in X} f(x, y) \leq \inf _{y \notin H} \max _{x \in K} f(x, y),
$$

then

$$
\inf _{y \in Y} \sup _{x \in X} f(x, y)=\sup _{x \in X} \inf _{y \in Y} f(x, y) .
$$

\section{REFERENCES}

Bayraktar, E., Huang, Y.-J. \& Zhou, Z. (2015), 'On hedging American options under model uncertainty', SIAM J. Financial Math. 6(1), 425-447.

URL: $h$ ttp://dx.doi.org/10.1137/140961869

Bayraktar, E. \& Zhou, Z. (2016a), 'Arbitrage, hedging and utility maximization using semi-static trading strategies with american options', Ann. Appl. Probab. 26(6), 3531-3558.

URL: http://dx.doi.org/10.1214/16-AAP1184

Bayraktar, E. \& Zhou, Z. (2016b), 'No-arbitrage and hedging with liquid American options', ArXiv e-prints .

Bouchard, B. \& Nutz, M. (2015), 'Arbitrage and duality in nondominated discrete-time models', Ann. Appl. Probab. 25(2), 823-859.

URL: http://dx.doi.org.proxy.lib.umich.edu/10.1214/14-AAP1011 
Deng, S. \& Tan, X. (2016), 'Duality in nondominated discrete-time models for Americain options', ArXiv e-prints .

Ha, C. W. (1981), 'A noncompact minimax theorem', Pacific J. Math. 97(1), 115-117.

URL: http://projecteuclid.org.proxy.lib.umich.edu/euclid.pjm/1102734660

Hobson, D. \& Neuberger, A. (2016), 'More on hedging American options under model uncertainty', available on ArXiv .

Hobson, D. \& Neuberger, A. (2017), 'Model uncertainty and the pricing of American options', Finance Stoch. 21(1), 285-329.

URL: $h t t p: / / d x$.doi.org/10.1007/s00780-016-0314-2

Neuberger, A. (2007), 'Bounds on the American Option', SSRN http://ssrn.com/abstract=966333

Department of Mathematics, University of Michigan

E-mail address: erhan@umich.edu

Institute for Mathematics And its Applications, University of Minnesota

E-mail address: zhouzhou@ima.umn.edu 